\title{
Dynamic Monte-Carlo modeling of hydrogen isotope reactive-diffusive transport in porous graphite
}

${ }^{*}$ R. Schneider ${ }^{a}$, A. Rai ${ }^{a}$, A. Mutzke ${ }^{a}$, M. Warrier ${ }^{b}$,E. Salonen ${ }^{c}$, K. Nordlund $^{d}$ ${ }^{a}$ Max-Planck-Institut für Plasmaphysik, D-17491 Greifswald, Germany

${ }^{b}$ Institute for Plasma Research, Gandhinagar, India

${ }^{c}$ Laboratory of Physics, HUT, P.O.Box 1100, FIN-02015, Finland

${ }^{d}$ Accelerator laboratory, P.O.Box 43, FIN-00014, University of Helsinki, Finland

* Corresponding author: Ralf Schneider

Corresponding author address:

HGF-NWG, Max-Planck-Institut für Plasmaphysik, Wendelsteinstrasse 1,

D-17491 Greifswald, Germany.

e-mail: ralf.schneider@ipp.mpg.de

Phone: +49 3834882400

Phone: +49 3834882409 


\title{
Dynamic Monte-Carlo modeling of hydrogen isotope reactive-diffusive transport in porous graphite
}

\author{
${ }^{*}$ R. Schneider ${ }^{a}$, A. Rai ${ }^{a}$, A. Mutzke ${ }^{a}$, M. Warrier ${ }^{b}$, E. Salonen ${ }^{c}$, K. Nordlund $^{d}$ \\ ${ }^{a}$ Max-Planck-Institut für Plasmaphysik, D-17491 Greifswald, Germany \\ ${ }^{b}$ Institute for Plasma Research, Gandhinagar, India \\ ${ }^{c}$ Laboratory of Physics, HUT, P.O.Box 1100, FIN-02015, Finland \\ ${ }^{d}$ Accelerator laboratory, P.O.Box 43, FIN-00014, University of Helsinki, Finland
}

\begin{abstract}
Deuterium and tritium will be the fuel used in a fusion reactor in equal mixtures. It is important to study the recycling and mixing of these isotopes of hydrogen in graphite from several points of view: (i) impact on the ratio of deuterium to tritium in a reactor, (ii) continued use of graphite as a first wall and divertor material, (iii) reaction with carbon atoms and the transport of hydrocarbons will provide insight into chemical erosion. Dynamic Monte-Carlo techniques are used to study the reactivediffusive transport of hydrogen isotopes and interstitial carbon atoms in a 3-D porous graphite structure irradiated with hydrogen and deuterium and is compared with published experimental results for hydrogen re-emission and isotope exchange.
\end{abstract}




\section{Introduction}

Deuterium and tritium will be the fuel used in a fusion reactor in equal mixtures. It is important to study the recycling and mixing of these isotopes of hydrogen in graphite from several points of view: (i) impact on the ratio of deuterium to tritium in a reactor, (ii) continued use of graphite as a first wall and divertor material, (iii) reaction with carbon atoms and the transport of hydrocarbons will provide insight into chemical erosion. The graphite used in fusion devices as a first wall material is porous and consists of granules and voids.

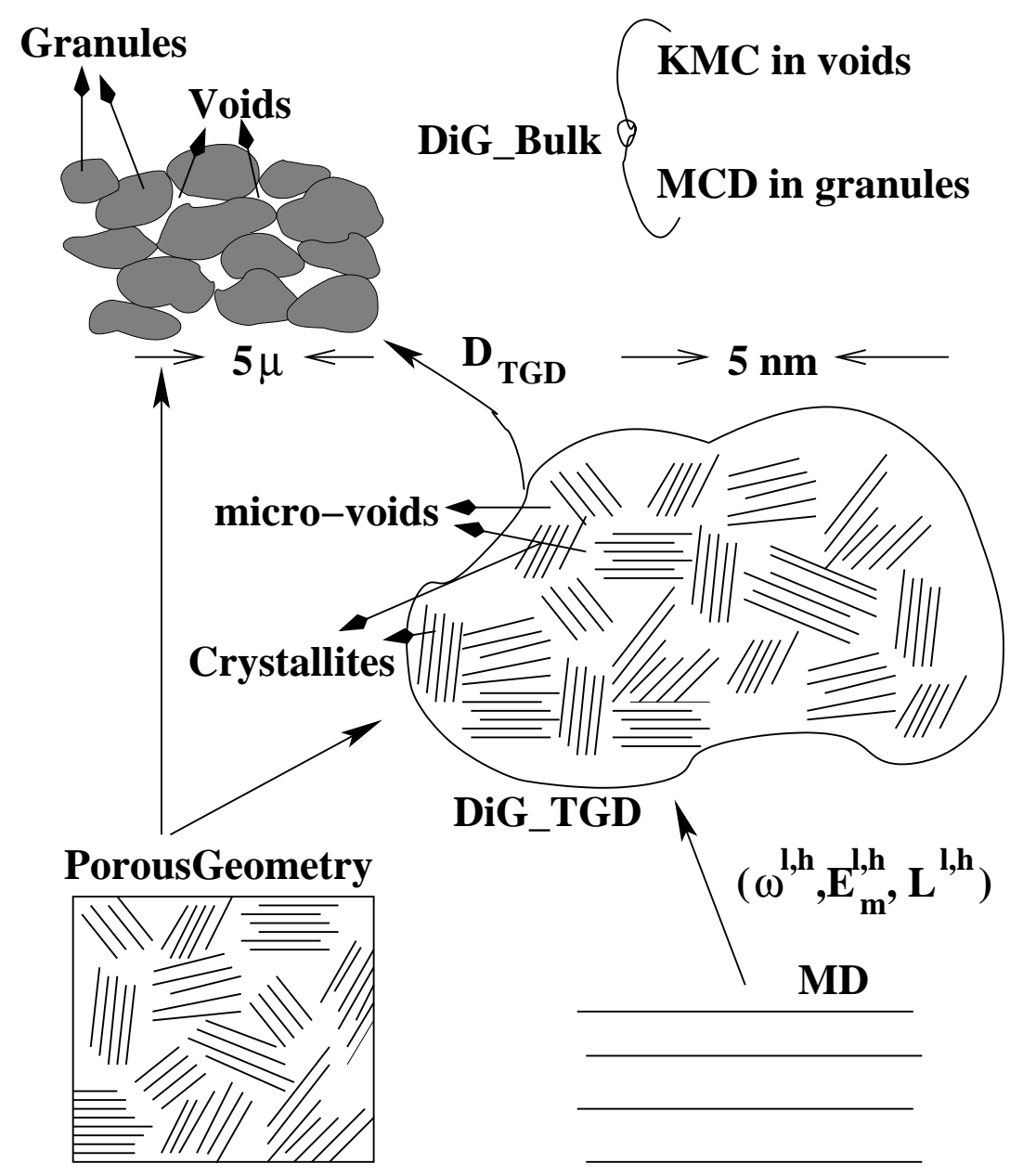

Fig. 1. Multi-scale schematic 
These granules are typically 1-10 micrometer separated by voids which are typically a fraction of a micrometer. The granules consist of graphitic micro-crystallites of size 10$100 \mathrm{~nm}$ separated by micro-voids which are typically one nm (Fig. 1) [1-4]. These substructures, voids and micro-voids provide a large internal surface area inside graphite where the interstitial atoms can diffuse and react with each other which will affect the hydrogen isotope inventory and recycling behaviour and also chemical erosion. It is estimated that the trap site concentrations within the graphite bulk are of the order $10^{-3}-10^{-5}[5,6]$. Graphites exposed to fusion edge plasmas also get damaged by the incident energetic ions and neutrals from the plasma and this causes a high density of trap sites within the range of penetration of the incident ions $([2,5]$ and references therein).

\section{Model}

We use dynamic Monte-Carlo techniques to study the reactive-diffusive transport of hydrogen isotopes and interstitial carbon atoms in a $3-\mathrm{D}$ porous graphite structure irradiated with hydrogen and deuterium and compare with published experimental results for isotope exchange.

Incident hydrogen ions and neutrals which are not reflected have a wide range of possibilities as discussed in $[1-3,7]$. They can get trapped either in the high density of traps region or in the bulk and become detrapped, or undergo chemical reactions to form hydrocarbons or recombine to form hydrogen molecules which can then diffuse 
along micro-voids or voids. They can be solute and become trapped, or diffuse within the crystallites, or diffuse along the crystallite surfaces or recombine to form molecules at the crystallite surfaces (or granule surfaces) and undergo diffusion along the microvoids (or voids).

The existence of such large variations in length scales of sub-structures coupled with the wide range of possible atomistic processes makes the study of hydrogen transport and inventory (or complementing this, the formation of hydrocarbons and their transport) in graphite a non trivial exercise. Many detailed macroscopic models have been proposed to study hydrogen isotope inventory and transport in porous graphite [1-4] and hydrocarbon formation and transport in graphite [8]. These models use rate constants for transport from experiments [9-13,7], some of which still need theoretical explanations. There exists many microscopic models [14-17] using MD with either empirical potentials or density functional theory and they give an insight into the microscopic mechanisms studied in graphite. It is desirable to use the insights gained from the microscopic models into modeling the transport in the meso-scale and further into the macro-scale in order to understand the physical processes contributing to macroscopic transport.

We have earlier modeled hydrogen isotope diffusion in pure, crystal graphite using MD at micro-scales $\left(2.5 \mathrm{~nm}, 10^{-10} \mathrm{~s}\right)$ and consistently parametrized the MD results within a KMC scheme [17]. A 3d, porous, granule structure was constructed using statistical distributions for crystallite dimensions and crystallite orientations for a specified micro-void fraction. The KMC scheme was extended to include trapping and 
detrapping at the crystallite-micro-void interface in the $3 \mathrm{~d}$ porous granule structure to simulate trans-granular-diffusion (TGD) in the meso-scales $\left(10^{-7}-10^{-6} \mathrm{~m}\right.$, several ms) [18] using the results from our micro-scale modeling and from experiments ([19] and references therein). Later, this concept was extended our simulations to the macroscales $(1 \mathrm{~cm}$, up to a few $s)$, thereby having a truly multi-scale capability.[20].

In this paper, we use an extension of this model by introducing in addition to the atomic also molecular species to study the reactive-diffusive transport of hydrogen isotopes and interstitial carbon atoms in a 3-D porous graphite structure irradiated with hydrogen and deuterium. We compare the model results with published experimental results for re-emission and isotope exchange.

The parametrization of the processes of the molecules is based on [21]. molecules in the crystallites can undergo a simple jump, characterized in terms of the KMC parameters migration energy $(2.0 \mathrm{eV})$, jump length $(0.3 \mathrm{~nm})$ and jump attempt frequency $(2.74$. $\left.10^{13} \mathrm{~s}^{-1}\right)$. Also, they can experience a dissociation $\left(4.4475 \mathrm{eV}, 0.2 \mathrm{~nm}, 2.74 \cdot 10^{13} \mathrm{~s}^{-1}\right)$. Within the voids we consider simple jumps or desorption $\left(0.06 \mathrm{eV}, 1.0 \mathrm{~nm}, 1.0 \cdot 10^{13} \mathrm{~s}^{-1}\right)$ or dissociation $\left(4.4475 \mathrm{eV}, 0.2 \mathrm{~nm}, 1.0 \cdot 10^{13} \mathrm{~s}^{-1}\right)$.

\section{Re-emission of hydrogen}

We initially create a cubic structure of $1 \times 10^{-7} \mathrm{~m}$ representing one typical granule. $200 \times 200 \times 200$ cells are used with an elementary size of $0.5 \mathrm{~nm}$. We consider a case of $2000 \mathrm{H}$ atoms uniformly distributed in X-Y at a depth of $3.75 \cdot 10^{-8} \mathrm{~m}$ along $\mathrm{Z}$ with 
a Gaussian distribution of width $7.8 \cdot 10^{-9} \mathrm{~m}$ as calculated from TRIM runs of $1 \mathrm{keV}$ hydrogen atoms impinging on carbon. Due to this short mean free path, modeling of one granule is sufficient for the re-emission studies.

In Fig. 2 the re-emitted flux for graphite with void fractions of $5 \%$ and $9 \%$ bombarded with $1 \mathrm{keV}$ hydrogen ions are shown.

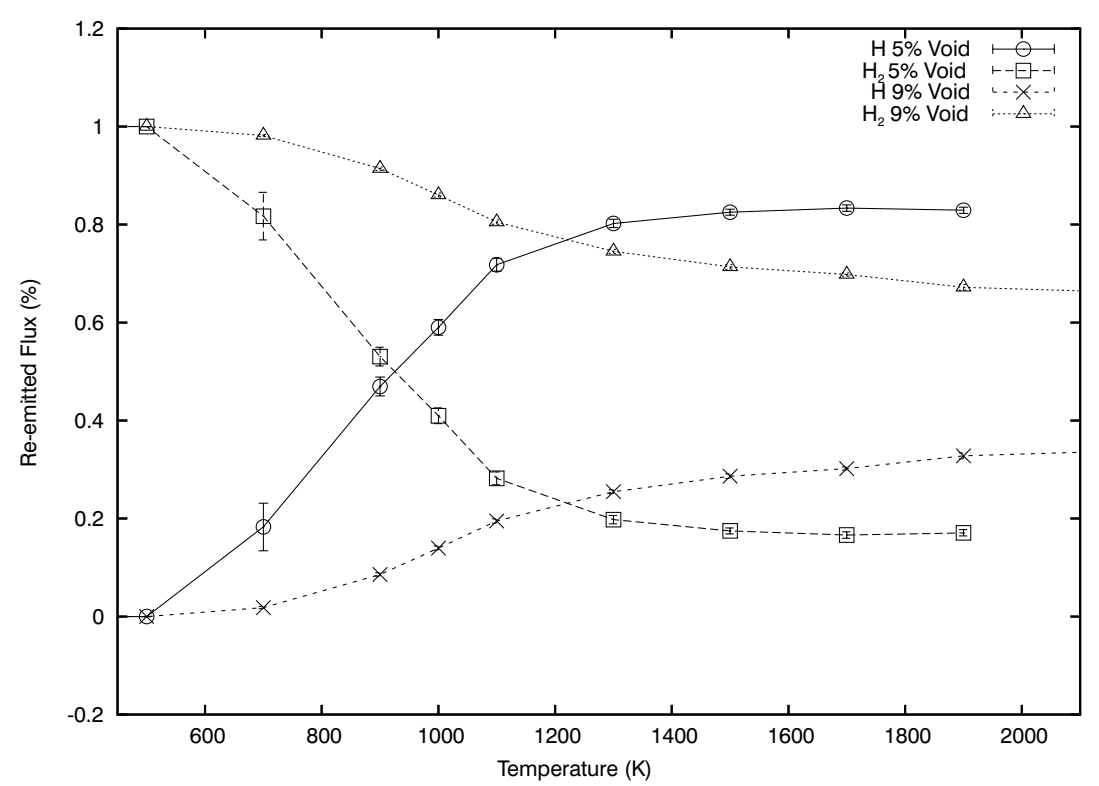

Fig. 2. Re-emitted flux of hydrogen atoms and molecules as a function of temperature for $5 \%$ and $99 \%$ voids. The elementary size of an void was in both cases $5 \cdot 10^{-9} \mathrm{~m}$.

The experimental results [22] are very well reproduced for the case of $5 \%$ void fraction in our model. The re-emission of atoms can be well explained following the model of Haasz et al. [2], where the re-emission process is basically governed by the competition between atom diffusion or desorption and recombination. The rise of the re-emission of atomic hydrogen at about $900 K$ is determined by the activation energy for entering the bulk. At these temperatures the diffusion within the granules is also quite low. Most of the transport is by surface diffusion of the solute atoms. Particles start getting into the bulk and from the bulk to the voids in our simulations at $1200 \mathrm{~K}$. 
Increasing the void fraction to $9 \%$ with constant elementary size, we observe that we get larger amounts of hydrogen molecules. As we increase the void fraction with same elementary void sizes we have more voids and lesser bulk elements, where a possible trapping of the atoms can happen (each bulk element is representing one micro-crystal where at its surface trapping/detrapping can occur). Therefore, more hydrogen molecule recombination events can occur and the molecular hydrogen flux increases (Fig. 3).

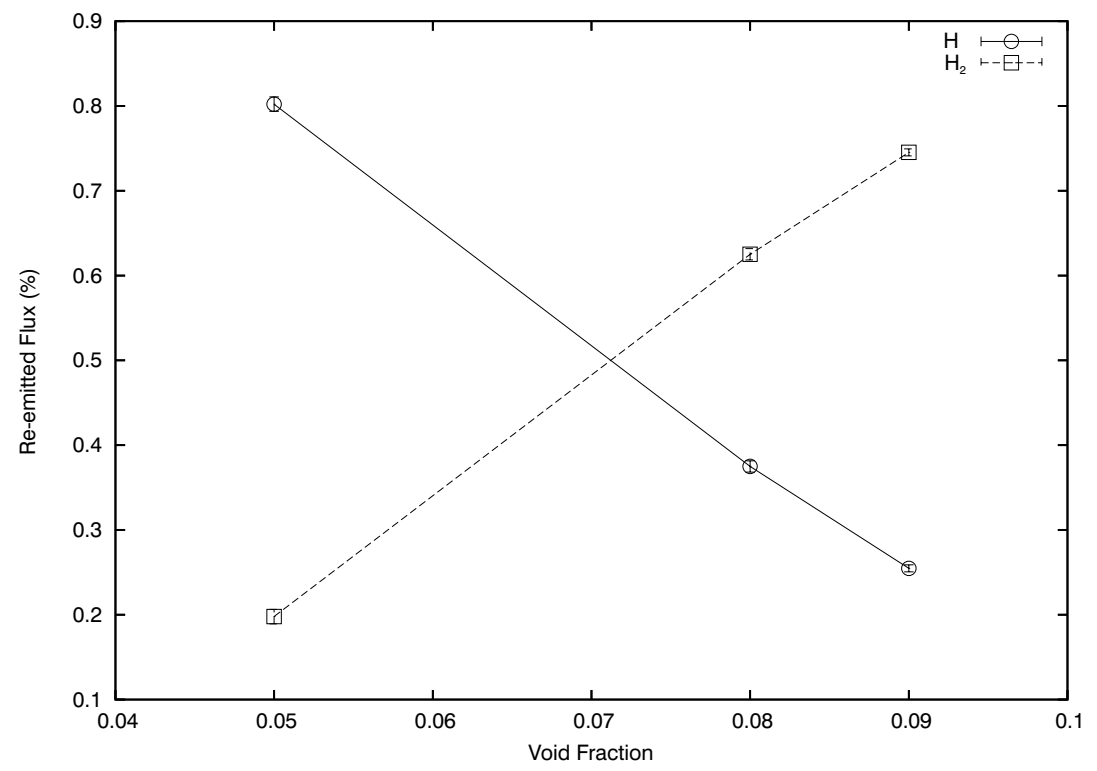

Fig. 3. Re-emitted flux of hydrogen atoms and molecules as a function of the void fraction at $1300 \mathrm{~K}$. The elementary size of the voids was $5 \cdot 10^{-9} \mathrm{~m}$.

Changing the elementary size and keeping the void fraction constant, Fig. 4 shows also an increase of the re-emitted hydrogen molecular flux with increasing elementary void size.

In this case, the system has smaller void surface with increasing elementary void sizes (small elementary voids create a large number of small voids, large elementary voids create a small number of large voids for a fixed void fraction). Therefore, less and 


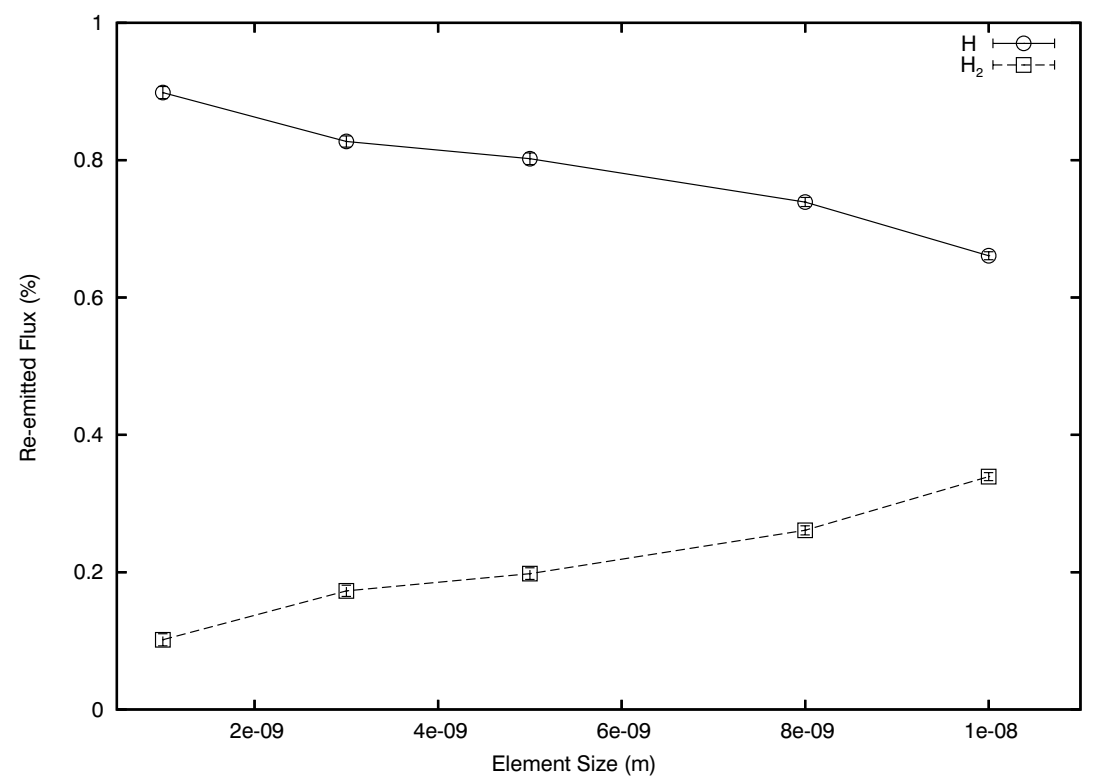

Fig. 4. Re-emitted flux of hydrogen atoms and molecules as a function of the elementary void size at $1300 \mathrm{~K}$ for a void fraction of $5 \%$.

less trapping/detrapping can occur at the void surfaces and more hydrogen molecular recombination events can happen.

\section{Isotope exchange}

For modelling of isotope exchange experiments with simultaneous ion beam bombardment of hyrdogen and deuterium $(1 \mathrm{keV}$ and $3 \mathrm{keV}$ with depth gaussian depthdistributions with mean values of $2.75 \cdot 10^{-8} \mathrm{~m}$ and $5.95 \cdot 10^{-8} \mathrm{~m}$ and standard deviations of $7.8 \cdot 10^{-9} \mathrm{~m}$ and $1.2 \cdot 10^{-8} \mathrm{~m}$ ). We used $280 \times 280 \times 280$ cells with an elementary cell size of $5.0 \cdot 10^{-10} \mathrm{~m}$ resulting in a system size of $1.4 \cdot 10^{-7} \mathrm{~m}$. Runs were done at a temperature of $1000 \mathrm{~K}$ and for a micro-void size of $6.0 \cdot 10^{-9} \mathrm{~m}$. Deuterium molecules in the crystallites can undergo a simple jump, characterized in terms of the KMC parameters migration energy $(2.0 \mathrm{eV})$, jump length $(0.3 \mathrm{~nm})$ 
and jump attempt frequency $\left(2.74 \cdot 10^{13} s^{-1}\right)$. Also, they can experience a dissociation $\left(4.5531 \mathrm{eV}, 0.2 \mathrm{~nm}, 2.74 \cdot 10^{13} \mathrm{~s}^{-1}\right)$. Within the voids we consider simple jumps or desorption $\left(0.06 \mathrm{eV}, 1.0 \mathrm{~nm}, 1.0 \cdot 10^{13} \mathrm{~s}^{-1}\right)$ or dissociation $(4.5531 \mathrm{eV}, 0.3 \mathrm{~nm}, 1.0$. $\left.10^{13} \mathrm{~s}^{-1}\right)$. HD molecules can also jump within crystallites $\left(2.0 \mathrm{eV}, 0.3 \mathrm{~nm}, 2.74 \cdot 10^{13} \mathrm{~s}^{-1}\right)$ or dissociate $\left(4.511 \mathrm{eV}, 0.2 \mathrm{~nm}, 2.74 \cdot 10^{13} \mathrm{~s}^{-1}\right)$ [21]. Within the voids they can jump or desorb $\left(0.06 \mathrm{eV}, 1.0 \mathrm{~nm}, 1.0 \cdot 10^{13} \mathrm{~s}^{-1}\right)$ or dissociate $\left(4.5106 \mathrm{eV}, 0.2 \mathrm{~nm}, 1.0 \cdot 10^{13} \mathrm{~s}^{-1}\right)$. The recombination distance for formation of molecules was taken as $0.2 \mathrm{~nm}[2]$.

For a void fraction of $7 \%$ steady-state conditions were reached after $1.2 \cdot 10^{-8} \mathrm{~s}$ (Fig. 5). The relative fluxes of the modelling agree well with experiments [23].

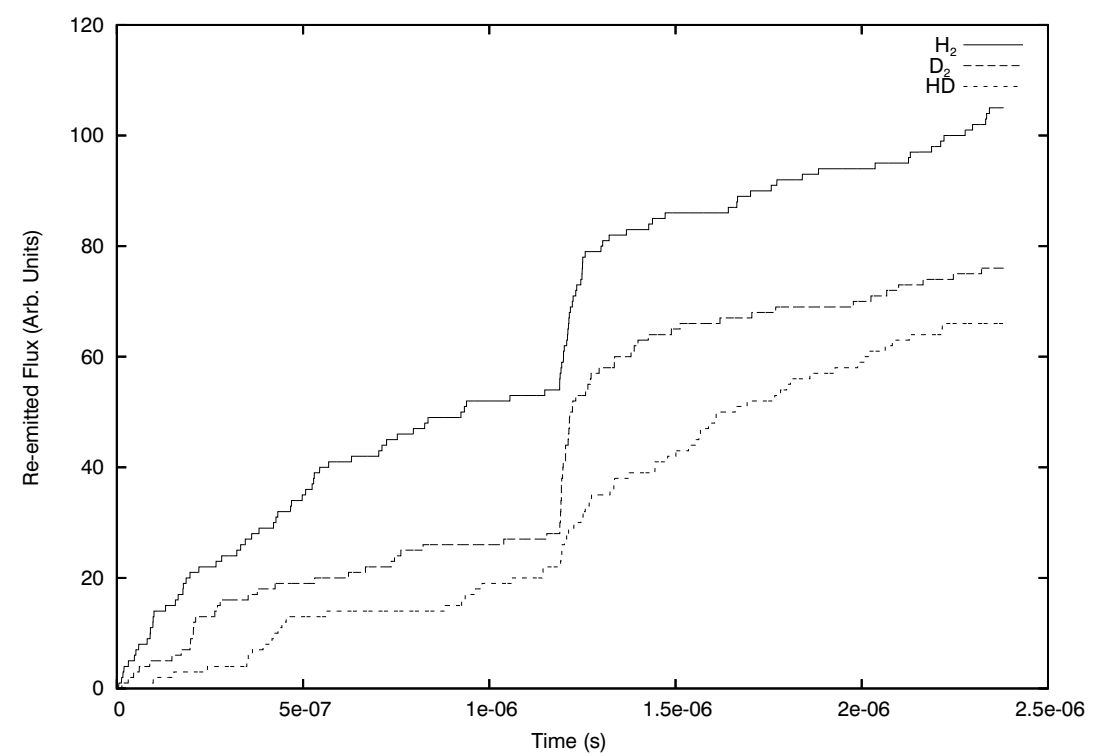

Fig. 5. Re-emitted flux of $H_{2}, D_{2}$ and $H D$ molecules as a function of time.

To test the idea, that the increase of hydrogen molecules observed experimentally at the beginning of the ion bombardment is due to micro-void creation, we continued the run with increasing the void fraction to $9 \%$. A rise of both the hydrogen and deuterium molecular fluxes is seen due to the increase of the void surfaces as discussed before. However, the experimentally observed jump of hydrogen molecular fluxes followed by 
a slow decay to steady state combined with the slow rise of deuterium molecular fluxes is not reproduced. In experiment, the ion-created microchannels are dominating the transport processes. In order to get a better agreement with the experiment, the dynamcis of this formation have to be included into the model. This is possible by adding to the time-step loop of our code one TRIM/TRIDYN calculation to determine the void distribution self-consistently in time.

\section{$5 \quad$ Summary and conclusions}

We have extended a multi-scale scheme to model hydrogen transport in porous graphite to molecule formation and transport. The influence of the size and fraction of voids for 3D porous graphite hydrogen re-emission was studied and agreed with existing models. The model should allow better quantative descriptions of these effects if the meso- and microstructure of the graphite is known. In case of isotope exchange an unresolved dicrepancy remains between modeling and experiment, because hydrogen and deuterium molecules show in modeling similar trends, whereas in experiment opposite time-depencies of these molecules after activating ion beams are observed. Inclusion of TRIM/TRIDYN calculations for self-consistent calculation of the void distributions can eventually overcome this. 


\section{Acknowledgments}

R. Schneider and A. Rai acknowledge funding of the work by the Initiative and Neworking Fund of the Helmholtz Association.

\section{References}

[1] Wolfhard Möller. J. Nucl. Mater., 162-164 (1989) 138.

[2] A. A. Haasz, P. Franzen, J. W. Davis, S. Chiu, and C. S. Pitcher. J. Appl. Phys., 77(1) $(1995) 66$.

[3] G. Federici and C.H. Wu. J. Nucl. Mater., 186 (1992) 131.

[4] A. Hassanein, B. Wiechers, and I. Konkashbaev. J. Nucl. Mater., 258-263 (1998) 295.

[5] M. Mayer, M. Balden, and R. Behrisch. J. Nucl. Mater., 252 (1998) 55.

[6] R. A. Causey, M. I. Baskes, and K. L. Wilson. J. Vac. Sci. Technol. A, 4(3) (1986) 1189.

[7] J. Küppers. Surface Science Reports, 22 (1995) 249.

[8] B. V. Mech, A. A. Haasz, and J. W. Davis. J. Appl. Phys., 84(3) (1998) 1655.

[9] B. M. U. Scherzer, M. Wielunski, W. Möller, A. Turos, and J. Roth. Nucl. Instrum. Methods Phys. Res. B, 33 (1988) 714.

[10] B. M. U. Scherzer, J. Wang, and W. Möller. J. Nucl. Mater., 162-164 (1989) 1013.

[11] W. Möller and B. M. U. Scherzer. J. Appl. Phys., 64 (1988) 4860.

[12] R. A. Causey. J. Nucl. Mater., 162-164 (1989) 151. 
[13] H. Atsumi, S. Tokura, and M. Miyake. Proc. 3rd Int. Conf. on Fusion Reactor Materials, Karlsruhe, (1987), J. Nucl. Mater., 155-157 (1988) 241.

[14] E. Salonen, K. Nordlund, J. Keinonen, and C. H. Wu. Phys. Rev. B, 63 (2001) 195415$(1-14)$.

[15] Y. Ferro, F. Marinelli, and A. Allouche. Chemical Physics Letters, 368 (2003) 609.

[16] Y. Ferro, F. Marinelli, and A. Allouche. Journal of Chemical Physics, 116 \# 18 (2002) 8124.

[17] M. Warrier, R. Schneider, E. Salonen, and K. Nordlund. Physica Scripta, accepted for publication (2003).

[18] M. Warrier, R. Schneider, E. Salonen, and K. Nordlund. Contrib. Plasma Phys., 44 \#1-3 (2004) 307.

[19] K. L. Wilson et. al. Atomic and plasma-material interaction data for fusion (supplement to the journal Nuclear Fusion, 1 (1991) 31.

[20] M. Warrier, R. Schneider, E. Salonen, and K. Nordlund. J. Nuclear Mat., in press.

[21] G. Herzberg. Phys. Rev. Lett., 23 (1969) 1081.

[22] P. Franzen and E. Vietzke. J. Vac. Sci. Technol. A, 12 \#3 (1994) 820.

[23] S. Chiu and A.A. Haasz. J. Nuclear Mat., 196-198 (1992) 972. 


\section{$7 \quad$ Figure captions}

Fig. 1: Multi-scale schematic

Fig. 2: Re-emitted flux of hydrogen atoms and molecules as a function of temperature for $5 \%$ and $99 \%$ voids. The elementary size of an void was in both cases $5 \cdot 10^{-9} \mathrm{~m}$.

Fig. 3: Re-emitted flux of hydrogen atoms and molecules as a function of the void fraction at $1300 \mathrm{~K}$. The elementary size of the voids was $5 \cdot 10^{-9} \mathrm{~m}$.

Fig. 4: Re-emitted flux of hydrogen atoms and molecules as a function of the elementary void size at $1300 K$ for a void fraction of $5 \%$.

Fig. 5: Re-emitted flux of $H_{2}, D_{2}$ and $H D$ molecules as a function of time. 
8 Figures

Granules
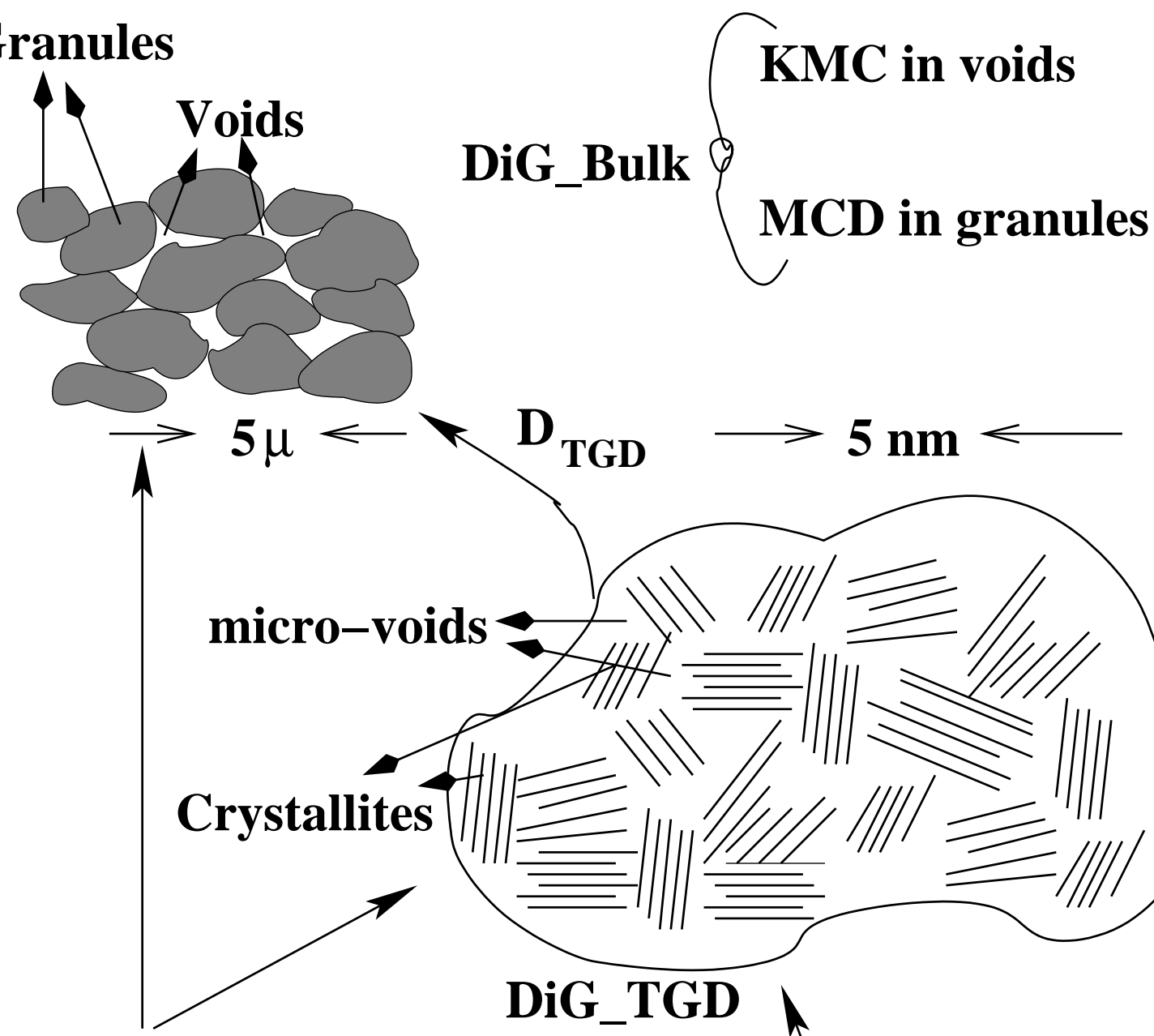

PorousGeometry

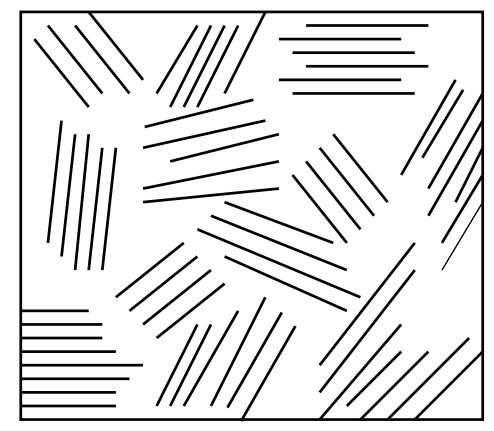

$\mathrm{D}_{\text {TGD }} \longrightarrow 5 \mathrm{~nm}<$
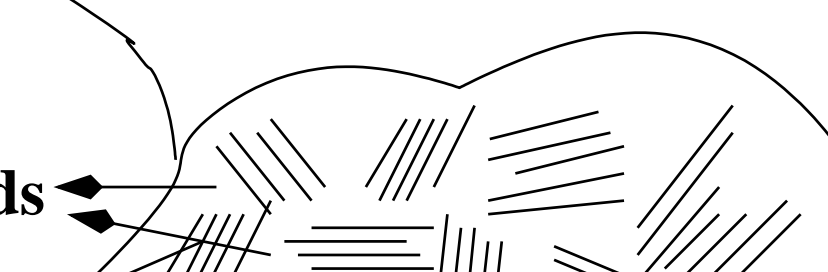

言
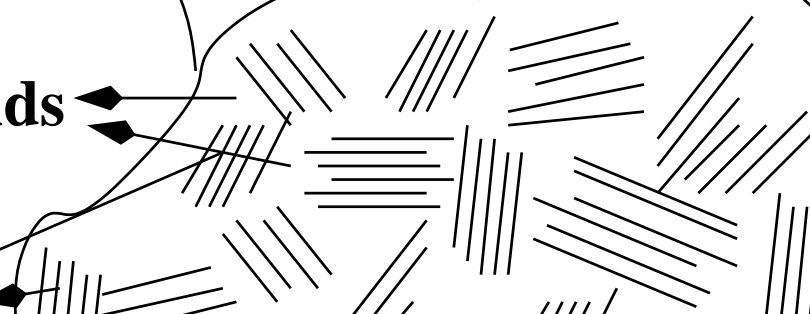

111

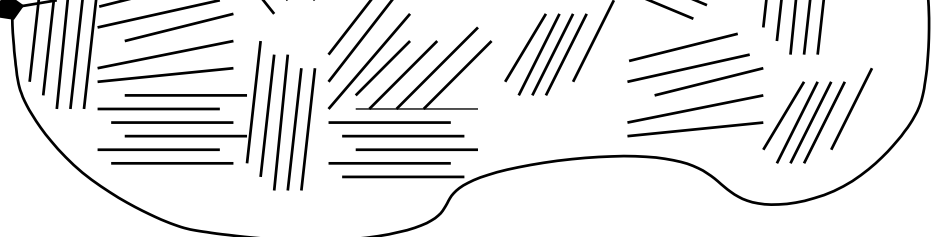

DiG_TGD

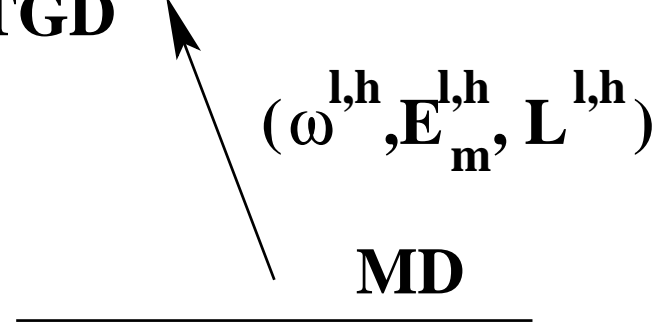



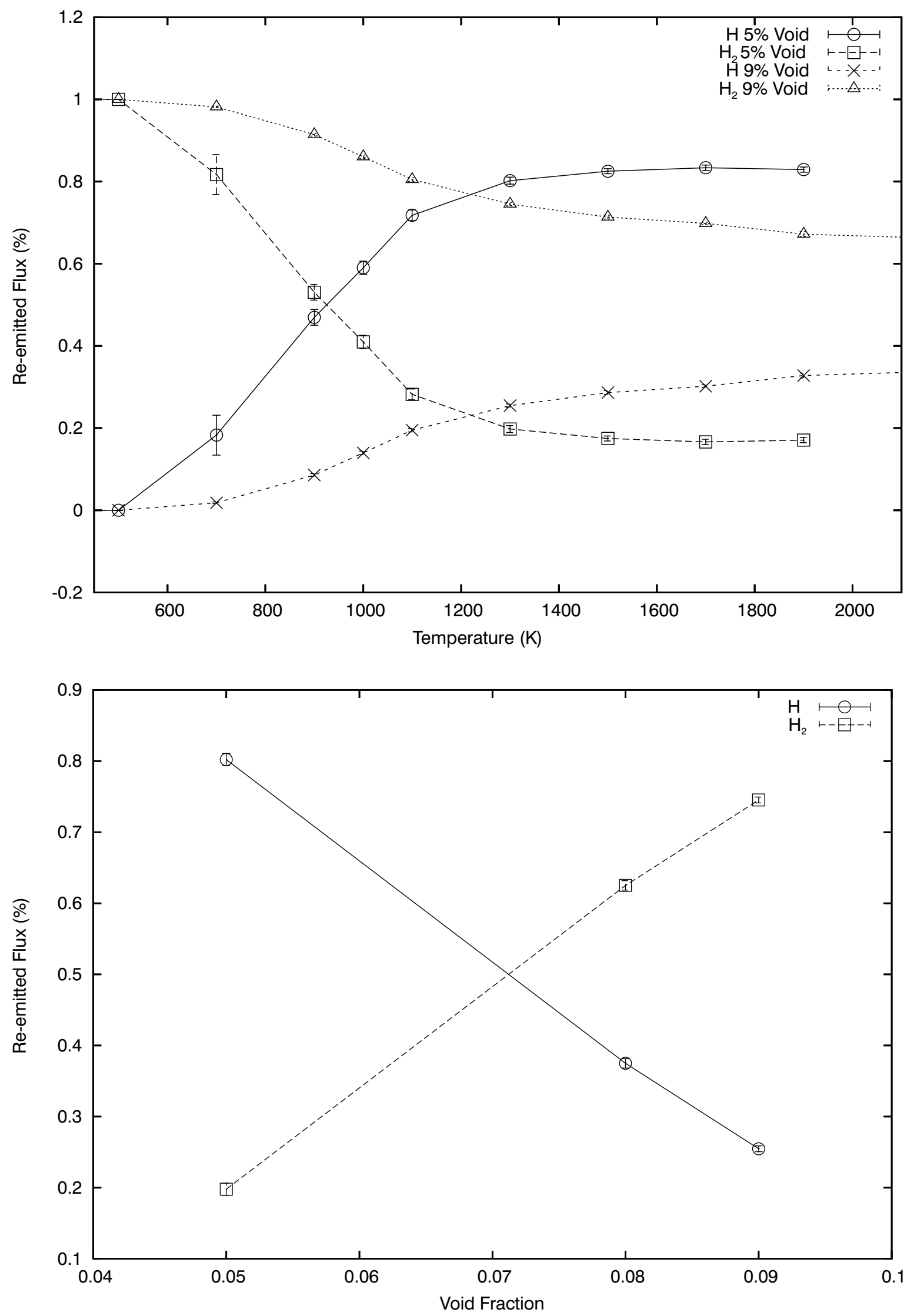

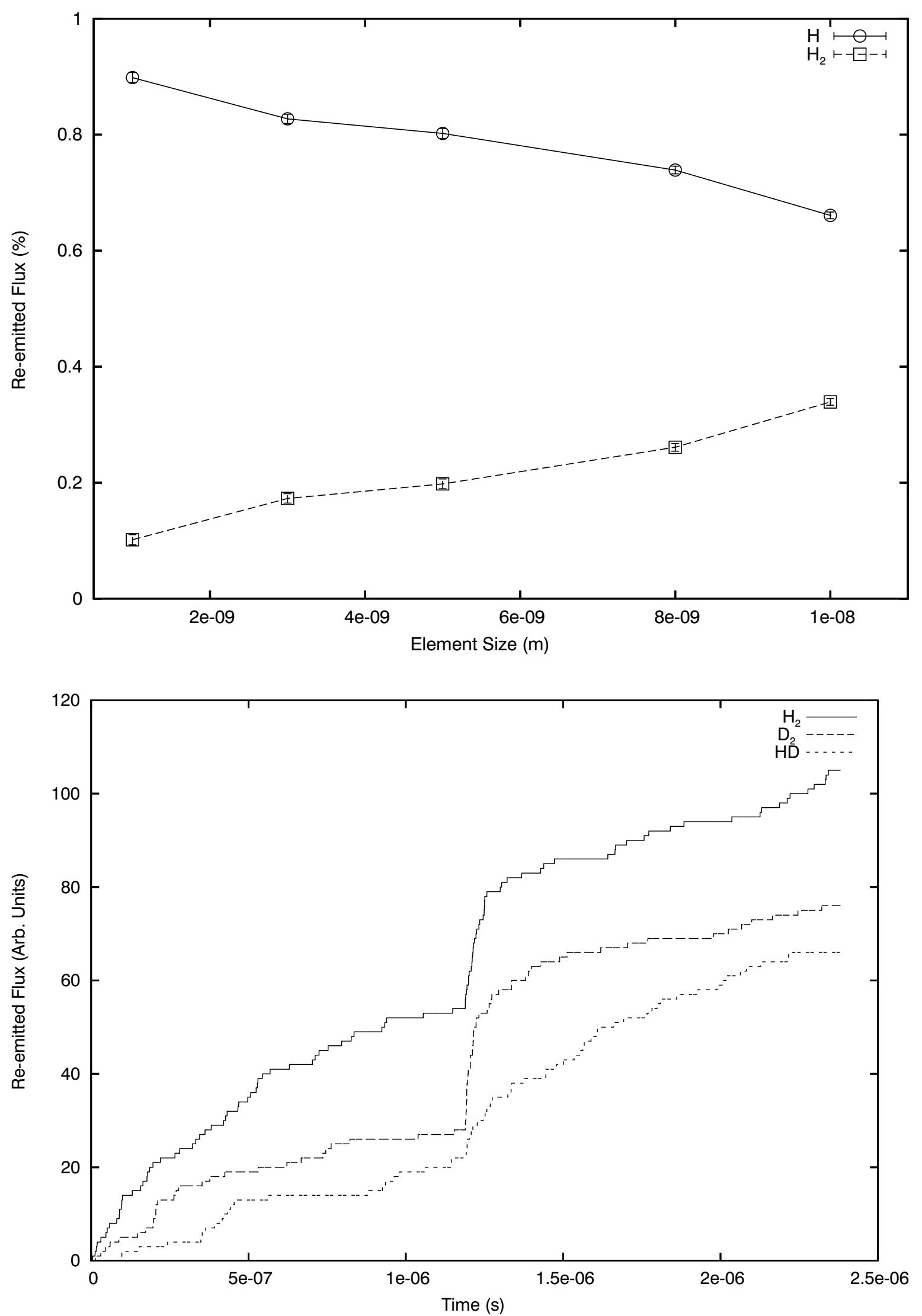